\title{
The Heat in Antiferromagnetic Switching
}

\author{
New experiments suggest that heat might be responsible for the current-induced voltage \\ signals measured in antiferromagnets, and not a rotation of the material's spins as \\ previously thought.
}

\section{by Barry Zink*}

$\int$ erromagnets - magnetic materials in which the spins of neighboring atoms align-form permanent magnets whose "sticky" properties have been exploited in technologies for millennia. But their close magnetic cousins the antiferromagnets, materials in which neighboring spins point in opposite directions, have yet to find practical use. This lack of use most likely arises from their pattern of alternating spins, which ties the magnetic flux up inside the material, making its magnetism difficult to detect and manipulate. Scientists are now developing so-called all-electrical methods to control antiferromagnets; these techniques may finally change this status quo. But new measurements from Chih-Chieh Chiang from National Taiwan University and colleagues highlight a problem with these methods, indicating that switching the pointing direc-

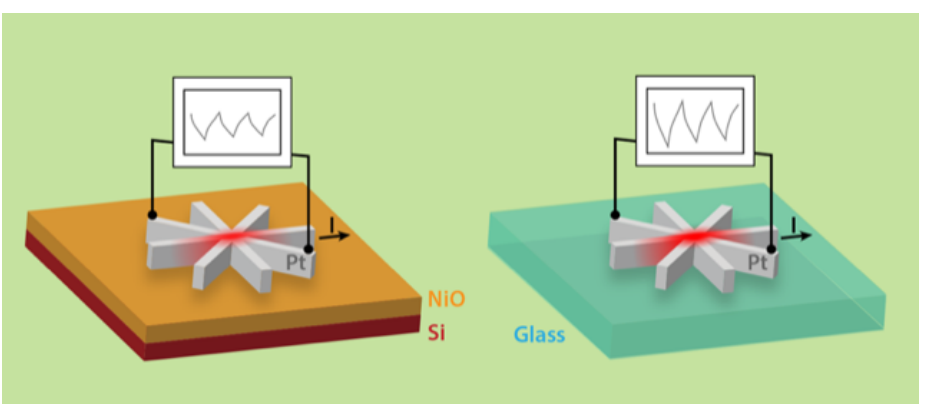

Figure 1: (Left) Platinum (Pt) strips grown on antiferromagnetic nickel oxide (NiO) films convert charge current to spin current, which is intended to switch the pointing direction of the insulating NiO's spins. The switching is observed via a sawtooth voltage pattern. However, the Pt heats dramatically when the current is applied and (right) this heating reproduces the sawtooth pattern even when no antiferromagnet is present. (APS/Alan Stonebraker)

* Department of Physics and Astronomy, University of Denver, Denver, CO, USA tions of an antiferromagnet's spins-a requirement for their use in devices-with all-electrical methods might be more difficult than researchers thought [1].

From the first iPod that stored music to today's server farms that house the world's many cat videos, the spins of ferromagnets-which encode data's 1's and 0's as domains of up- and down-pointing spins-have been key to making usable devices for storing information. The success of this technology has led researchers to ask whether spin might also be utilized for processing information. To achieve that, so-called spintronic circuits, which can carry spin currents, would likely need to become smaller, more stable, and faster than they are today. That is where antiferromagnetic materials come in. For data storage, their pattern of alternating spins reduces the impact of stray or nearby magnetic fields, potentially reducing data loss by making their bits more stable. For spin transport, the antiferromagnetic order of the spins should increase the maximum speed at which a spin wave can carry magnetic information through the material, allowing devices made from antiferromagnets to operate faster than those made from ferromagnets.

Several recent advances have brought antiferromagnets closer to practical use. In 2014, researchers demonstrated spin transport through an insulating antiferromagnet. The achievement could allow for faster and more energy efficient devices than current devices made from metals or semiconductors, as running a spin current through an insulator causes less heating [2]. More critically, in 2016, researchers discovered a simple way to electrically "switch" an antiferromagnetic bit [3]. Switching involves rotating the axes of the spins by some angle and is important for writing data.

To electrically switch a magnetic domain, one injects a current of conduction electrons. The intrinsic angular momentum, or spin, of these electrons interacts with the orbital angular momentum of the atoms in the material, generating a flow of spins called a spin current. If this spin current is absorbed by the magnetic domain it causes a torque that can switch the domain. This switching mechanism is therefore called spin-orbit torque (SOT) switching. SOT switching is a powerful tool for manipulating ferromagnets [4], and there are many tools for confirming a switch in these materials. 
The same is not true for antiferromagnets, where proving that a bit has switched turns out to be more difficult. To observe the switching in metallic antiferromagnets the authors of Ref. [3] adapted an electrical measurement that is sensitive to the ordering of the material's spins. Specifically, they applied a series of large current pulses to the metallic antiferromagnet with consecutive pulses flowing in opposite directions. Switching was then monitored via the voltage induced by the planar Hall effect. A sawtooth pattern in the voltage was taken as confirmation of antiferromagnetic switching.

Very shortly after this groundbreaking work on a metallic antiferromagnet, research groups across the globe applied the technique to antiferromagnetic insulators, such as nickel oxide $(\mathrm{NiO})$. In these experiments, the insulator is interfaced to a metallic layer, most often platinum $(\mathrm{Pt})$. A charge current in the $\mathrm{Pt}$ generates a spin current that can flow into the $\mathrm{NiO}$ and switch regions of the antiferromagnet's spins. Researchers initially relied on the same electrical detection scheme used for metallic antiferromagnets, and indeed they saw the characteristic sawtooth pattern. But the new work demonstrates that this key electrical signature can also be generated in a device with no magnetic components at all, leading to questions about the technique's efficacy for detecting switching.

Chiang and colleagues started by depositing 4-nm-thick Pt strips in a star configuration on top of a 60-nm-thick NiO film grown on a silicon substrate (Fig. 1). This device reproduced those commonly used for $\mathrm{Pt} / \mathrm{NiO}$ SOT switching experiments. Applying charge current pulses of about 30 $\mathrm{mA}$ that were intended to generate spin currents that would reorient the $\mathrm{NiO}^{\prime}$ s spins, the team observed the characteristic sawtooth voltage pattern. In a second device, the researchers replaced the silicon with glass, a poor conductor of heat, and observed a large increase in the magnitude of the sawtooth voltage. This result is unexpected, since the $\mathrm{NiO}$ layer was nominally similar in both experiments. Finally, in a third device they deposited the Pt directly on glass, removing the magnetic material entirely (Fig. 1). The sawtooth signal remained and had an even larger magnitude than either of the signals detected for the other two devices. This dramatic result clearly shows that the electrical signal observed in these experiments cannot be evidence of switching of the insulating antiferromagnet.

Using computer models, Chiang and colleagues make a strong case that the sawtooth voltage pattern is instead caused by heat generated in the Pt strips. This heat drives thermoelectric voltages that persist for surprisingly long periods of time, even up to one hour. The warmed $\mathrm{Pt}$ and the resulting thermal gradients that develop between the various Pt strips depend on the heat conductivity of the underlying substrate, which is why glass, with its low heat conductivity, causes larger voltages. Thermal gradients of this sort frequently arise in spintronic devices and offer chal- lenges and opportunities in their use [5].

Questions remain regarding the details of the physical mechanism generating the sawtooth signals, which could involve electromigration - the transport of electrons due to the presence of an electric field. Chiang et al.'s work raises concerns that we must resolve to realize spintronic devices based on insulating antiferromagnets. However, the researchers may paint with too broad a brush when they question whether SOT switching has been demonstrated in antiferromagnets at all. This concern ignores a good deal of evidence in favor of antiferromagnetic switching, including the demonstration of a memory element using CuMnAs, a metallic antiferromagnet [6], and of imaging techniques that show modification of antiferromagnetic domains in response to charge currents [7-12]. Techniques ranging from synchrotron $x$-ray measurements to novel thermal scanning probe microscopy have also provided clear proof of switching in both metallic and insulating antiferromagnets, though never with the uniform domain reversal seen in ferromagnets [4]. All of these other techniques are much slower to implement than electrical ones, so measurements are made long after the heating caused by "writing" currents has dissipated.

Chiang et al.'s work could explain a puzzling aspect of the switching experiments. A large voltage signal is often detected from what imaging techniques show is quite minor realignment of the antiferromagnetic domain pattern. Perhaps the nonmagnetic heating effect that Chiang and colleagues observe provides the signal's origin. If so, that would potentially resolve this mystery. The team also highlights a number of other puzzles that need to be solved to continue progress in the field. For example, does an antiferromagnetic film that is only a few nanometers thick have the same spin structure as the bulk material? Do the different interfaces, which arise from varying the substrate, modify the spin-orbit coupling throughout the film stack? Perhaps most importantly, does an artifact-free electrical method exist for detecting antiferromagnetic switching in insulators? These questions highlight the difficulty in harnessing antiferromagnets, but answering them could yet put antiferromagnets to very practical use.

This research is published in Physical Review Letters.

\section{REFERENCES}

[1] C. C. Chiang, S. Y. Huang, D. Qu, P. H. Wu, and C. L. Chien, "Absence of evidence of electrical switching of the antiferromagnetic Néel vector," Phys Rev. Lett. 123, 227203 (2019).

[2] H. Wang, C. Du, P. C. Hammel, and F. Yang, "Antiferromagnonic spin transport from $\mathrm{Y}_{3} \mathrm{Fe}_{5} \mathrm{O}_{12}$ into NiO," Phys. Rev. Lett. 113, 097202 (2014).

[3] P. Wadley et al., "Electrical switching of an antiferromagnet," Science 351, 587 (2016).

[4] A. Manchon, J. Železný, I. M. Miron, T. Jungwirth, J. Sinova, A. Thiaville, K. Garello, and P. Gambardella, "Current-induced 
spin-orbit torques in ferromagnetic and antiferromagnetic systems," Rev. Mod. Phys. 91, 035004 (2019).

[5] C. H. Back, G. E. W. Bauer, and B. L. Zink, "Special issue on spin caloritronics," J. Phys. D. Appl. Phys. 52, 230301 (2019).

[6] K. Olejník et al., "Antiferromagnetic CuMnAs multi-level memory cell with microelectronic compatibility," Nat. Comun. 8, 15434 (2017).

[7] M. J. Grzybowski et al., "Imaging current-induced switching of antiferromagnetic domains in CuMnAs," Phys. Rev. Lett. 118, 057701 (2017).

[8] P. Wadley et al., "Current polarity-dependent manipulation of antiferromagnetic domains," Nat. Nanotech. 13, 362 (2018).

[9] S. Yu. Bodnar, M. Filianina, S. P. Bommanaboyena, T. Forrest, F. Maccherozzi, A. A. Sapozhnik, Y. Skourski, M. Kläui, and M.
Jourdan, "Imaging of current induced Néel vector switching in antiferromagnetic Mn $\mathrm{M}_{2} \mathrm{Au}$," Phys. Rev. B 99, 140409R (2019).

[10] T. Moriyama, K. Oda, T. Ohkochi, M. Kimata, and T. Ono, "Spin torque control of antiferromagnetic moments in NiO," Sci. Rep. 8, 14167 (2018).

[11] L. Baldrati et al., "Mechanism of Néel order switching in antiferromagnetic thin films revealed by magnetotransport and direct imaging," Phys. Rev. Lett. 123, 177201 (2019).

[12] I. Gray et al., "Spin Seebeck imaging of spin-torque switching in antiferromagnetic Pt/NiO heterostructures," Phys. Rev. X 9, 041016 (2019).

10.1103/Physics. 12.134 